\title{
Comparison of methods for the assessment of nonlinearity in short-term heart rate variability under different physiopathological states
}

Cite as: Chaos 29, 123114 (2019); https://doi.org/10.1063/1.5115506

Submitted: 18 June 2019 . Accepted: 19 November 2019 . Published Online: 12 December 2019

Luca Faes (D), Manuel Gómez-Extremera (D), Riccardo Pernice (D), Pedro Carpena (D), Ciandomenico Nollo, Alberto Porta (iD), and Pedro Bernaola-Galván

\section{AIP Conference Proceedings}

FLASH WINTER SALE!

$\mathbf{5 0 \%}$ OFF ALL PRINT PROCEEDINGS

ENTER CODE 50DEC19 AT CHECKOUT 


\title{
Comparison of methods for the assessment of nonlinearity in short-term heart rate variability under different physiopathological states
}

\author{
Cite as: Chaos 29, 123114 (2019); doi: 10.1063/1.5115506 \\ Submitted: 18 June 2019 . Accepted: 19 November 2019. \\ Published Online: 12 December 2019
}

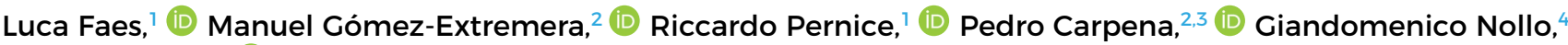
Alberto Porta, 5,6 (ID) and Pedro Bernaola-Galván ${ }^{2,3}$

\author{
AFFILIATIONS \\ 1'Department of Engineering, University of Palermo, 90128 Palermo, Italy \\ ${ }^{2}$ Dpto. de Física Aplicada II, ETSI de Telecomunicación, University of Málaga, 29071 Málaga, Spain \\ ${ }^{3}$ Instituto Carlos I de Física Teórica y Computacional, University of Málaga, 29071 Málaga, Spain \\ ${ }^{4}$ Department of Industrial Engineering, University of Trento, 38123 Trento, Italy \\ ${ }^{5}$ Department of Biomedical Sciences for Health, University of Milan, 20122 Milan, Italy \\ ${ }^{6}$ Department of Cardiothoracic, Vascular Anesthesia and Intensive Care, IRCCS Policlinico San Donato, 20097 San Donato \\ Milanese, Milan, Italy
}

\begin{abstract}
Despite the widespread diffusion of nonlinear methods for heart rate variability (HRV) analysis, the presence and the extent to which nonlinear dynamics contribute to short-term HRV are still controversial. This work aims at testing the hypothesis that different types of nonlinearity can be observed in HRV depending on the method adopted and on the physiopathological state. Two entropy-based measures of time series complexity (normalized complexity index, NCI) and regularity (information storage, IS), and a measure quantifying deviations from linear correlations in a time series (Gaussian linear contrast, GLC), are applied to short HRV recordings obtained in young (Y) and old (O) healthy subjects and in myocardial infarction (MI) patients monitored in the resting supine position and in the upright position reached through head-up tilt. The method of surrogate data is employed to detect the presence and quantify the contribution of nonlinear dynamics to HRV. We find that the three measures differ both in their variations across groups and conditions and in the percentage and strength of nonlinear HRV dynamics. $\mathrm{NCI}$ and IS displayed opposite variations, suggesting more complex dynamics in $\mathrm{O}$ and MI compared to Y and less complex dynamics during tilt. The strength of nonlinear dynamics is reduced by tilt using all measures in Y, while only GLC detects a significant strengthening of such dynamics in MI. A large percentage of detected nonlinear dynamics is revealed only by the IS measure in the Y group at rest, with a decrease in $\mathrm{O}$ and MI and during T, while NCI and GLC detect lower percentages in all groups and conditions. While these results suggest that distinct dynamic structures may lie beneath short-term HRV in different physiological states and pathological conditions, the strong dependence on the measure adopted and on their implementation suggests that physiological interpretations should be provided with caution.
\end{abstract}

Published under license by AIP Publishing. https://doi.org/10.1063/1.5115506

Historically, the study of heart rate variability (HRV) has both received clinical attention, e.g., as a tool for risk stratification after myocardial infarction, and has attracted the interest of physicists who saw it as a particularly lucid example of chaos in physiology. Later on, after it was realized that a thorough evaluation of the chaotic nature of cardiac dynamics is precluded by difficulties inherent in the noisy nature of biological signals and in the restricted length of the data typically available, the field of HRV analysis underwent a shift in paradigm from chaos to complexity (intended as unpredictability) and detection of nonlinear dynamics in short-term HRV (up to a few minutes of recordings) analyzed in different pathophysiological states. The latter issue remains elusive, due to the difficulty of reliably assessing nonlinearity over short time series, to the proliferation of diverse nonlinear analysis methods, each with its own strengths and limitations, and to the changing nature of nonlinear HRV dynamics across states and conditions. The present study contributes to settling this issue, implementing different state-of-the-art nonlinear dynamic measures and comparing them in regard to the detection of the presence and the contribution of nonlinear dynamics to short-term HRV. The comparison is performed considering the progression across healthy and pathological states (i.e., aging and myocardial infarction) and investigating the effects on the cardiac dynamics of a specific physiological stressor (i.e., head-up tilt). 


\section{INTRODUCTION}

Human heart rate variability (HRV), commonly assessed measuring the spontaneous beat-to-beat changes in the duration of the RR interval of the electrocardiogram (ECG), is the result of the activity of different physiological control systems, which operate across multiple time scales to let the body functions adapt to environmental, physical, and psychological challenges. ${ }^{1,7} \mathrm{RR}$ interval fluctuations have been classically represented as a linear superposition of rhythms, ${ }^{2}$ leading to remarkable time- and frequency-domain descriptions of the factors contributing to the neuroautonomic modulation of the heart rhythm in healthy conditions, as well as of the alteration of these factors related to a variety of pathological states. . $, 8,18,22,39,42$ Nevertheless, since the cardiac control is typically accomplished through the interaction among multiple complex regulatory mechanisms, including self-sustained oscillators as well as control loops, ${ }^{31}$ the linear description of the RR interval variability may be severely limited and disregard significant dynamical features.

As a consequence, a variety of nonlinear approaches to time series analysis have been devised to characterize RR interval fluctuations and extract additional physiological and clinical information from HRV. ${ }^{60,64}$ A class of these approaches, focused on long-term analyses spanning scales up to several hours, is mainly based on using methods able to assess scaling properties, long-range correlations, and multifractality of the RR time series. ${ }^{5,24,30,46}$ These nonlinear methods were often employed with the aim of identifying signatures typical of chaotic dynamics in long-term HRV recordings, leading to an animated discussion of this topic. ${ }^{19}$ Besides the presence or absence of chaos, ${ }^{14,21,28}$ there is a substantial consensus about the fact that long-term RR interval time series are nonlinear and multifractal and that the scaling behavior of HRV is altered with aging or during physical exercise and under pathological conditions such as myocardial infarction. ${ }^{6,20,23,25,29}$

On the other hand, it is also widely accepted that the assessment of HRV over temporal scales ranging from seconds to a few minutes allows the indirect investigation of the mechanisms underlying the short-term cardiovascular control, ${ }^{13,41}$ and this assessment might require nonlinear methods better suited for the evaluation of complex aspects of HRV dynamics. In fact, a number of nonlinear measures have been developed to this end, e.g., based on nonlinear prediction, ${ }^{53,57}$ entropy or mutual information, ${ }^{50,66}$ time irreversibility, ${ }^{54,62}$ or phase coupling. ${ }^{3,12}$ These and other studies have provided ample evidence that changes in nonlinear descriptors of short-term HRV such as complexity or regularity indexes, either induced by the modification of the experimental conditions or determined by spontaneous transitions among physiological states, can be reliably detected and associated with alterations of the autonomic control. Notwithstanding this, the presence and impact of nonlinear dynamics in short-term HRV is still a controversial issue. Some studies suggested that nonlinear components of HRV are of limited importance in resting conditions and are evoked by the presence of a dominant respiratory sinus arrhythmia ${ }^{53,57}$ or in association with respiratory inputs to the cardiovascular system. ${ }^{17,26}$ Conversely, other studies assessing temporal asymmetries suggested that nonlinearities are relevant at rest and may be present even in conditions of small respiratory sinus arrhythmia. ${ }^{54}$ The contribution of the two branches of the autonomic nervous system to nonlinear HRV dynamics remains elusive and is likely linked to the time scales of their functioning. ${ }^{52}$ Moreover, nonlinear dynamics might be sustained by the interaction between sympathetic and parasympathetic activities.

Methodologically, it has been suggested that multiple nonlinear components, operating at different scales and possibly interacting with each other, may concur to the generation of shortterm HRV. ${ }^{3,27,52}$ Since these different components of HRV nonlinear dynamics may be captured in a different way by different metrics, the aim of the present study is to test the hypothesis that distinct types of nonlinear dynamics underlie HRV during different physiopathological states. To this end, we apply three nonlinear dynamic measures to the RR interval time series measured in young and old healthy subjects, as well as in postacute myocardial infarction (AMI) patients, monitored at rest and during sympathetic activation induced by postural change. The measures were selected, among those allowing the assessment of nonlinear dynamics in short-term HRV ( $\sim 300$ points), to investigate such dynamics from different perspectives, exploiting different concepts and implementing different estimators. In particular, two of the measures quantify the common concepts of complexity and regularity previously defined in time series analysis with the indexes of sample entropy ${ }^{58}$ and information storage, ${ }^{38}$ implementing them through refined estimation techniques devised recently. ${ }^{51,66}$ The third measure is defined building upon several previous works ${ }^{9,11,34-37}$ to quantify the deviation from linearity of the correlation structure of the observed time series according to a recently proposed Gaussian Linear Contrast method (GLC). ${ }^{10}$ The application of these approaches in conjunction with the method of surrogate data ${ }^{59,61}$ allows us to quantify the extent to which nonlinear dynamics impact on short-term HRV in different conditions of autonomic nervous system imbalance, also investigating the effects of age and pathology. The database used in the study is made publicly available to favor reproducibility and encourage the comparison with different nonlinear dynamic measures.

\section{NONLINEAR DYNAMIC MEASURES}

This section describes the methods used in the present work to quantify nonlinear dynamics in the temporal statistical structure of a system evolving in time. Our starting point is an experimental time series $\left\{s_{n}\right\}, n=1,2, \ldots, N$, which is considered as a realization of a stochastic process $S$ describing the evolution over time of an observed dynamical system $\mathcal{S}$. The process $S$ is considered stationary so that the random variables obtained sampling the process at the time $n$ (i.e., $\left.S_{n}, n=1,2, \ldots, N\right)$ are identically distributed with marginal probability density function $f(\cdot)$ and cumulative density function $F(\cdot)$. Moreover, without loss of generality, we assume that each $S_{n}$ has zero mean and unit standard deviation.

To assess nonlinear dynamics in the stochastic process $S$, we look at its temporal correlation structure: while for purely linear dynamics the dependence between $s_{n}$ and $s_{n-\ell}$ is linear for any lag $\ell$, in the case of nonlinear dynamics such dependence cannot be studied only in terms of linear correlations. In the first two methods considered, nonlinear correlations are investigated within an information-theoretic framework, separating the present state of the system $S_{n}$ from its past states $S_{n}^{-}=\left[S_{1}, \ldots, S_{n-2}, S_{n-1}\right]$ and quantifying their information content in terms of entropy measures. ${ }^{66}$ In fact, when the system transits from past states to a new state, new 
information is produced in addition to the information that is already carried by the past states. The rate of generation of new information is inversely related to the strength of nonlinear correlations in the process, while the information shared between the present and the past variables is directly related to such correlations. ${ }^{66}$ On this basis, the measures of conditional entropy (Sec. II A) and information storage (Sec. II B) assess nonlinear correlations quantifying, respectively, the new information contained in $S_{n}$ but not in $S_{n}^{-}$and the amount of information carried by $S_{n}$ that can be explained by $S_{n}^{-}$.

The third method takes its roots on the observation that a purely linear stochastic process is considered to have only linear correlations. However, the most feasible models for generation of pure linear processes give a Gaussian marginal distribution as output so that linear processes with marginal Gaussian distributions are usually considered as reference for linearity. This can lead to false positive cases by nonlinearity methods when applied to linear processes with non-Gaussian marginal distribution. In the GLC (see Sec. II C), we test nonlinearity based only on the nature of correlations, which truly reflect the dynamics and discard those effects induced by non-Gaussian marginal distributions.

\section{A. Complexity index based on local sample entropy}

The information-theoretic assessment of nonlinear correlations in a dynamic process is based on applying the concepts of entropy and conditional entropy to the random variables representing the present and past states of the process. Given two generic continuous (possibly vector) random variables $X$ and $Y$, the entropy of $X$ and the conditional entropy of $X$ given $Y$ are defined as

$$
\begin{aligned}
& H(X)=-\mathbb{E}[\log f(x)]=-\int_{\mathcal{D}_{X}} f(x) \log f(x) d x, \\
& H(X \mid Y)=-\mathbb{E}[\log f(x \mid y)]=H(X, Y)-H(Y),
\end{aligned}
$$

where $\mathcal{D}_{X}$ is the domain of $X, f(x)$ and $f(x \mid y)$ are the probability density of $X$ and the conditional probability of $X$ given $Y$, and $\mathbb{E}[\cdot]$ is the expectation operator; the term $H(X, Y)$ in $(2)$ is the joint entropy of $X$ and $Y$, obtained generalizing (1) to the joint probability density $f(x, y)=f(x \mid y) f(y)$. Particularizing these definitions to the variables $S_{n}$ and $S_{n}^{-}$describing the present and the past states of the process $S$, the conditional entropy becomes

$$
H\left(S_{n} \mid S_{n}^{-}\right)=H\left(S_{n}^{-}, S_{n}\right)-H\left(S_{n}^{-}\right) .
$$

The conditional entropy quantifies the amount of information contained in the present of the process that cannot be explained by its past history: if the process is fully random, the system produces information at the maximum rate, yielding maximum conditional entropy; if, on the contrary, the process is fully predictable, the system does not produce new information and the conditional entropy is zero.

In the present work, practical computation of the conditional entropy is performed adopting kernel estimates of the probability density functions. ${ }^{66}$ In particular, we make use of the well known sample entropy index, ${ }^{58}$ improved through the implementation of a local version of the estimator. ${ }^{51}$ The Sample Entropy (SampEn) estimates $H\left(S_{n} \mid S_{n}^{-}\right)$in (3), first truncating $S_{n}^{-}$to $S_{n}^{m}=\left[S_{n-1}, S_{n-2}, \ldots, S_{n-m}\right]$ and then approximating $H\left(S_{n}^{m}, S_{n}\right)$ and
$H\left(S_{n}^{m}\right)$ as the negative logarithm of the average joint probability of finding a pattern in the neighborhood of the reference pattern with a tolerance $r$ in the $(m+1)$-dimensional and $m$-dimensional embedding space, namely,

$$
\operatorname{SampEn}(m, r, n)=-\ln \left\langle p\left(S_{n}^{m}, S_{n}\right)\right\rangle+\ln \left\langle p\left(S_{n}^{m}\right)\right\rangle,
$$

where $p\left(S_{n}^{m}, S_{n}\right)$ is the probability that the pattern $S_{n}^{m} \oplus S_{n}$ assumes the value $s_{n}^{m} \oplus s_{n}$ and $p\left(S_{n}^{m}\right)$ is the probability that the pattern $S_{n}^{m}$ takes the value $s_{n}^{m}$ and $\langle\cdot\rangle$ performs the average over time (i.e., over all values $s_{n}^{m} \oplus s_{n}$ ). SampEn is a robust estimator of irregularity given that the log-of-zero situation is extremely unlikely because the logarithm is applied to the average of a quantity that has 0 as the lowest bound. However, as a consequence of computing an average over time, Sam$\mathrm{pEn}$ has the disadvantage to be a global marker of irregularity that might not represent reliably the local behavior in the neighborhood of a specific pattern and blur nonlinear features. ${ }^{51} \mathrm{~A}$ local version of SampEn (LSampEn) was proposed in Ref. 51 by directly approximating $H\left(S_{n} \mid S_{n}^{m}\right)$ instead of its constituents [i.e., $H\left(S_{n}^{m}, S_{n}\right)$ and $\left.H\left(S_{n}^{m}\right)\right]$ as

$$
\operatorname{LSampEn}(m, r, n)=-\ln \left\langle p\left(S_{n} \mid S_{n}^{m}\right)\right\rangle,
$$

where $p\left(S_{n} \mid S_{n}^{m}\right)$ is the conditional probability that the current state $S_{n}$ assumed the value $s_{n}$ given that the past state $S_{n}^{m}$ is $s_{n}^{m}$. The average operator makes the estimator robust against the log-of-zero situation and the estimation of $p\left(S_{n} \mid S_{n}^{m}\right)$ renders LSampEn a local estimator of irregularity given that the quantity being averaged referred specifically to the reference pattern $S_{n}^{m}$. To limit the consequence that, when solely $S_{n}^{m}$ is found in the neighborhood of $S_{n}^{m}, p\left(S_{n} \mid S_{n}^{m}\right)$ is unreliably high, ${ }^{49}$ we applied the correction proposed by Porta et al. $;^{51}$ namely, in this unfortunate case, $p\left(S_{n} \mid S_{n}^{m}\right)$ is set to $(N-m+1)^{-1}$ corresponding to the maximum uncertainty computable over the series. The resulting estimator, applied to the time series reduced to unit variance, is denoted as Normalized Complexity Index (NCI).

\section{B. Regularity index based on information storage}

Information measures can be exploited also for evaluating in a direct way the strength of nonlinear correlations in the dynamical structure of a stochastic process so that to assess its degree of regularity. To this end, a relevant entropy measure is the so-called information storage, which quantifies the amount of information shared between the present and the past observations of the considered process. The information storage of the process $S$ is defined as

$$
I\left(S_{n} ; S_{n}^{-}\right)=H\left(S_{n}\right)+H\left(S_{n}^{-}\right)-H\left(S_{n}^{-}, S_{n}\right),
$$

where $I(\cdot ; \cdot)$ denotes mutual information. The information storage reflects the degree to which information is preserved in a timeevolving system. ${ }^{65}$ As such, it measures how much of the uncertainty about the present can be resolved by knowing the past: if the process is fully random, the past gives no knowledge about the present, so that the information storage is zero; if, on the contrary, the process is fully predictable, the present can be fully predicted from the past, which results in maximum information storage. Note that information storage and conditional entropy of a dynamic process are inversely related to each other and depend on the entropy of the present state of the process through the equation $I\left(S_{n} ; S_{n}^{-}\right)+H\left(S_{n} \mid S_{n}^{-}\right)=H\left(S_{n}\right)$. 
In practical analysis, the information storage can be estimated from a time series of finite length following the same principles of conditional entropy estimation. These include the use of a finite number of samples in the past to approximate the history of the observed process (i.e., $S_{n}^{-}$is truncated to $S_{n}^{m}=\left[S_{n-1}, S_{n-2}, \ldots, S_{n-m}\right]$ ) and the adoption of nonparametric estimators of the probability density functions involved in the computation of $I\left(S_{n} ; S_{n}^{m}\right)$. However, since computation of the measure defined in (6) requires to estimate three entropy terms involving variables of different dimensions and since the bias of entropy estimates depends strongly on the dimension, implementation of standard histograms or kernel-based methods typically results in inaccurate estimates of the information storage. ${ }^{15,66}$ Here, while the kernel-based estimation of information storage is implemented for comparative purposes in the supplementary material, to overcome this limitation, we resort to nearest-neighbor entropy estimation ${ }^{32}$ and implement a strategy for bias compensation specific of mutual information estimates. ${ }^{3}$ The nearest-neighbor entropy estimate of a generic $d$-dimensional random variable $X$ can be obtained from a set of realizations $\left\{x_{1}, x_{2}, \ldots, x_{N}\right\}$ of the variable as ${ }^{32}$

$$
H(X)=\psi(N)-\psi(k)+d\left\langle\ln \varepsilon_{n}\right\rangle,
$$

where $\psi$ is the digamma function, $\varepsilon_{n}$ is twice the distance between the outcome $x_{n}$ and its $k$ th nearest neighbor computed according to the maximum norm (i.e., taking the maximum distance of the scalar components), and $\langle\cdot\rangle$ stands for average over $N$ outcomes. Then, the information storage could be computed applying (7) to the three terms in (6). However, doing so would result in different distance lengths when approximating the probability density in different dimensions, and this would introduce different estimation biases that cannot be compensated by taking the entropy differences. To keep the same distance length in all explored spaces, we perform a neighbor search only in the highest-dimensional space (the one spanned by the realizations of $S_{n}^{m}, S_{n}$ ) and then project the distances found in this space to the lower-dimensional spaces (those spanned by the realizations of $S_{n}^{m}$ and $S_{n}$ ), keeping these distances as the range within which neighbors are counted. Specifically, the knn estimate of $H\left(S_{n}^{m}, S_{n}\right)$ is computed through a neighbor search, i.e., fixing the number of neighbors $k$ and computing the distance to the $k$ th neighbor, as

$$
H\left(S_{n}, S_{n}^{m}\right)=\psi(N)-\psi(k)+(m+1)\left\langle\ln \varepsilon_{n}\right\rangle,
$$

where $\varepsilon_{n}$ is twice the distance from $\left(S_{n}, S_{n}^{m}\right)$ to its $k$ th nearest neighbor and then, given the distances $\varepsilon_{n}$, the entropies in the lowerdimensional spaces are estimated through a range search, i.e., fixing the distance $\varepsilon_{n}$ and counting for the neighbors falling within this distance, as

$$
\begin{gathered}
H\left(S_{n}^{m}\right)=\psi(N)-\psi\left(N_{S_{n}^{m}}\right)+m\left\langle\ln \varepsilon_{n}\right\rangle, \\
H\left(S_{n}\right)=\psi(N)-\psi\left(N_{S_{n}}\right)+\left\langle\ln \varepsilon_{n}\right\rangle,
\end{gathered}
$$

where $N_{S_{n}}$ and $N_{S_{n}^{m}}$ are the number of points whose distance from $S_{n}$ and $S_{n}^{m}$, respectively, is smaller than $\varepsilon_{n} / 2$. Finally, our estimate of the information storage is obtained subtracting Eq. (8) from the sum of Eqs. (9) and (10),

$$
I S=\psi(N)+\psi(k)-\left\langle\psi\left(N_{S_{n}^{m}}\right)\right\rangle-\left\langle\psi\left(N_{S_{n}}\right)\right\rangle .
$$

\section{Nonlinearity index based on Gaussian linear contrast}

As we stated above, GLC assesses nonlinearities related only to the nature of the correlations and not to the non-Gaussianity of the data. Let us consider an experimental time series $\left\{s_{n}\right\}$ $(n=1,2, \ldots, N)$, with non-Gaussian marginal distribution. The observed autocorrelation function of $\left\{s_{n}\right\}$ is given by

$$
C_{\text {obs }}(\ell)=\left\langle s_{n} s_{n+\ell}\right\rangle \text {. }
$$

Using $C_{\mathrm{obs}}(\ell)$, GLC tries to determine if $\left\{s_{n}\right\}$ is originated from a Gaussian time series $\left\{z_{G, n}\right\}(n=1,2, \ldots, N)$ with only linear correlations, which have been transformed to have the observed marginal distribution using an invertible transformation of the experimental time series. If this is the case, then GLC assumes that $\left\{s_{n}\right\}$ is linear and is nonlinear otherwise.

The theoretical background of the GLC method is the following. Let us consider a pair of correlated Gaussian variables $x_{G}$ and $y_{G}$, both of $\mathcal{N}(0,1)$ type so that their corresponding probability density and cumulative distribution are the standard Gaussian $\varphi\left(x_{G}\right)$ and $\Phi\left(x_{G}\right)$. We assume that $x_{G}$ and $y_{G}$ are only linearly correlated, with a correlation value $C_{G}$, i.e.,

$$
C_{G}=\left\langle x_{G} y_{G}\right\rangle \text {. }
$$

This is equivalent to affirm that the joint distribution of $x_{G}$ and $y_{G}$ is the bivariate Gaussian distribution $\varphi_{2}\left(x_{G}, y_{G}, C_{G}\right)$. Then, we transform $x_{G}$ and $y_{G}$ to the variables $x$ and $y$, which follow the marginal distribution of the experimental time series. This can be done with the usual method,

$$
x=F^{-1}\left[\Phi\left(x_{G}\right)\right], \quad y=F^{-1}\left[\Phi\left(y_{G}\right)\right],
$$

with $F^{-1}(\cdot)$ being the inverse cumulative distribution of the experimental time series. Since $F^{-1}$ is fixed by $\left\{s_{i}\right\}$, the linear correlation $C$ between $x$ and $y$, i.e., $C=\langle x y\rangle$ depends solely on the $C_{G}$ value. Indeed, since $x$ and $y$ depend formally on $x_{G}$ and $y_{G}$ [Eq. (14)] with joint distribution $\varphi_{2}\left(x_{G}, y_{G}, C_{G}\right), C$ can be calculated as (see Refs. 9-11 and 34-37)

$$
\begin{aligned}
C\left(C_{G}\right) \equiv\langle x y\rangle= & \int_{-\infty}^{\infty} \int_{-\infty}^{\infty} F^{-1}\left(\Phi\left(x_{G}\right)\right) F^{-1}\left(\Phi\left(y_{G}\right)\right) \varphi_{2} \\
& \times\left(x_{G}, y_{G}, C_{G}\right) d y_{G} d x_{G} .
\end{aligned}
$$

Solving numerically the previous integral for a dense set of $C_{G}$ values in the interval $(-1,1)$, we characterize the $C\left(C_{G}\right)$ function, which contains the information on how the Gaussian correlations are transformed when the distribution of the variables is transformed from Gaussian to the experimental distribution.

These results can be extrapolated straightforwardly to time series. Let us consider a Gaussian time series $\left\{z_{G, n}\right\}$, with autocorrelation function $C_{G}(\ell)$ given by $C_{G}(\ell) \equiv\left\langle z_{G, n} z_{G, n+\ell}\right\rangle$. Note that $z_{G, n}$ and $z_{G, n+\ell}$ are equivalent to $x_{G}$ and $y_{G}$ in Eq. (13). Then, let us transform $\left\{z_{G, n}\right\}$ into a time series $\left\{z_{n}\right\}$ with the same marginal distribution of the experimental time series using Eq. (14) for each $z_{G, n}$ value. The autocorrelation function $C(\ell)$ of $\left\{z_{n}\right\}$ can be then calculated using Eq. (15) simply by replacing $x_{G}, y_{G}$, and $C_{G}$ by $z_{G, n}, z_{G, n+\ell}$ and $C_{G}(\ell)$, respectively. In other words, once the $C\left(C_{G}\right)$ function is known by using Eq. (15) (which only requires the marginal distribution of the 
experimental time series), then $C(\ell)=C\left(C_{G}(\ell)\right)$. This last equality holds if the non-Gaussian time series $\left\{z_{n}\right\}$ really comes via the transformation (14) from the Gaussian and linearly correlated series $\left\{z_{G, n}\right\}$ since this is the condition used in Eq. (15) to determine $C\left(C_{G}\right)$. This property is the key point in the GLC method.

With this theoretical background, the steps to apply the GLC method on an experimental time series $\left\{s_{n}\right\}$ are the following:

(i) Determine the observed autocorrelation function $C_{\text {obs }}(\ell)$ of the experimental time series $\left\{s_{n}\right\}$.

(ii) Transform $\left\{s_{n}\right\}$ to have Gaussian distribution using the inverse of the transformation in Eq. (14) and calculate its autocorrelation function $C_{G^{\prime}}(\ell)$. Note that if $\left\{s_{n}\right\}$ has been obtained from a Gaussian time series using the transformation (14), simply by inverting the transformation the hypothetical original Gaussian time series is recovered, as long as the transformation from Gaussian to $\left\{s_{n}\right\}$ is invertible. The knowledge of $C_{\mathrm{obs}}(\ell)$ and $C_{G^{\prime}}(\ell)$ for each $\ell$ allows obtaining the function $C_{\text {obs }}\left(C_{G^{\prime}}\right)$.

(iii) Obtain the real $C\left(C_{G}\right)$ function using Eq. (15) by giving to $C_{G}$ a great number of values in the interval $(-1,1)$. In practice, and especially for short experimental time series, the numerical solution of the integral might be a harsh task: due to finite size effects, it can be difficult to correctly estimate $F^{-1}$. Then, to calculate $C\left(C_{G}\right)$, we adopt a different strategy: we use autoregressive time series of order 1 (AR1) with the same size as $\left\{s_{n}\right\}$. An AR1 process is defined as $z_{G, n}=\varphi z_{G, n-1}+\eta_{n}$, where $\left\{\eta_{n}\right\}$ is a Gaussian $\mathcal{N}(0,1)$ white noise and $\varphi \in(-1,1)$ is a constant. AR1 processes are Gaussian with purely linear correlations, with generic autocorrelation $C_{G}(\ell)=\varphi^{\ell}$, so that changing the $\varphi$ value, we can obtain any value of Gaussian correlation in the interval $(-1,1)$. Thus, when generating a large set of AR1 time series for different $\varphi$ values and calculate the autocorrelation function of all of them, we would obtain a huge amount of data points densely populating the $(-1,1)$ Gaussian correlation interval. Nevertheless, in order to save computation time, in practice, after preliminary analysis of $C_{\mathrm{obs}}\left(C_{G^{\prime}}\right)$, we restrict the $\varphi$ values to those that will generate Gaussian correlation values in our region of interest. Then, we transform all AR1 time series using Eq. (14) to have the marginal distribution of $\left\{s_{n}\right\}$ and also calculate the autocorrelation function $C(\ell)$ for all series. Note that each $C(\ell)$ value is the image of a Gaussian autocorrelation value. Finally, we bin the Gaussian correlation interval $(-1,1)$ into 0.01 length bins and put in each one the images of all the Gaussian correlation values contained in the bin. The average of all the images in the respective bin gives the $C$ value corresponding to the Gaussian correlation at the center of the bin so that finally, we have a numeric determination of the $C\left(C_{G}\right)$ function.

(iv) If the experimental time series $\left\{s_{n}\right\}$ is really obtained by transforming a Gaussian time series, then the Gaussian series is the one determined in step (ii), with autocorrelation function $C_{G^{\prime}}(\ell)$, and the observed autocorrelation is given by $C_{\text {obs }}(\ell)=$ $C_{\text {obs }}\left(C_{G^{\prime}}(\ell)\right)$. However, the expected correlations in $\left\{s_{n}\right\}$ if the Gaussian series is purely linear, $C_{\operatorname{lin}}(\ell)$, should be given by the $C\left(C_{G}\right)$ function determined in step (iii) evaluated at the $C_{G^{\prime}}(\ell)$ values, i.e., $C_{\operatorname{lin}}(\ell)=C\left(C_{G^{\prime}}(\ell)\right)$. The series $\left\{s_{n}\right\}$ is linear when $C_{\text {obs }}(\ell)=C_{\operatorname{lin}}(\ell)$ and is not linear otherwise. In this way, to quantify the nonlinearity of $\left\{s_{n}\right\}$, we can define the GLC nonlinearity index as

$$
G L C \equiv \sum_{\ell=1}^{\ell_{\mathrm{m}}}\left|C_{\text {obs }}(\ell)-C_{\text {lin }}(\ell)\right| .
$$

Actually, this GLC value measures the deviation of the observed dependence from that of a Gaussian process. Note that if the Gaussian process is transformed to a different marginal distribution (the one of the observed time series) using an invertible transformation, the dependence of the values is not modified since the transformation preserves the ordering of the values in the time series. In this case, GLC will be very close to zero, and then large GLC values will indicate nonlinearity. However, if the observed time series comes from a noninvertible transformation of a Gaussian process, GLC will be larger than zero despite the linear underlying dynamics so that this situation could be considered as a false positive. Nevertheless, for noninvertible transformations, the dependence of the time series will be different from that of a Gaussian process, since the transformation will change the ordering of the values. In this sense, we could say that the observed time series presents a higher complexity than a Gaussian process and will be reflected also in the GLC value.

In summary, GLC measures any deviation from the dependence in a Gaussian process, the paradigm of the simplest correlated process. According to this, GLC could be considered as a complexity measure, although strictly speaking, GLC measures nonlinearity only when the signal is originated with an invertible transformation. This approach is similar to that used in the copula theory ${ }^{43}$ when the dependence is studied avoiding the effects of the particular marginal distribution.

\section{DETECTION AND QUANTIFICATION OF NONLINEARITY}

\section{A. Detection of nonlinear dynamics}

For each individual analyzed HRV time series, we first assess the presence of nonlinear dynamics exploiting the method of surrogate data. ${ }^{61}$ This approach is based on (i) a null hypothesis to be rejected; (ii) a surrogate data set constructed in accordance with the null hypothesis; (iii) a discriminating statistic that has to be calculated on original and surrogate series; and (iv) a statistical test allowing to reject or confirm the null hypothesis.

The null hypothesis set in our case is that the investigated time series is a realization of a Gaussian stochastic process (fully described by linear temporal autocorrelations), eventually measured through a static and possibly nonlinear transformation distorting the Gaussian distribution.

The surrogate time series were generated in order to preserve the linear autocorrelation structure as well as the marginal distribution of the original time series. This was achieved through the iteratively refined amplitude adjusted Fourier Transform (IAAFT) method. ${ }^{59}$ The method is an improvement of the Fourier Transform (FT) method, ${ }^{61}$ which generates surrogate time series by computing the FT of the original series, substituting the Fourier phases with random numbers uniformly distributed between 0 and $2 \pi$, and finally 
performing the inverse FT. Since the FT method distorts the amplitude distribution of the original process when such a distribution is not Gaussian, the IAAFT method is followed implementing an iterative procedure that alternatively constrains the surrogate series to have the same power spectrum (by replacing the squared Fourier amplitudes of the candidate surrogate series with those of the original series) and to have the same amplitude distribution (by a rank ordering procedure) of the original series.

As discriminating statistic, we employ each of the three nonlinear indexes presented in Sec. II, i.e., the normalized complexity index (NCI) based on local sample entropy, the regularity index based on information storage (IS), and the nonlinear index based on Gaussian Linear Contrast (GLC).

As a statistical test, we perform a nonparametric test based on percentiles. The test compares the selected nonlinear index, here denoted generically as $N I$, when calculated on the original time series $\left(N I_{o}\right)$ and when calculated on $n_{s}$ surrogate time series $\left(n_{s}=100\right.$ in this work) generated under the null hypothesis. Specifically, $N I_{o}$ was compared with a threshold for significance $N I_{\alpha}$ extracted from the empirical distribution of $N I$ over the surrogates setting a prescribed confidence level $\alpha$ ( $\alpha=0.05$ in this work). In the case of the NCI index measuring the complexity of a time series, the index is expected to decrease in the presence of nonlinear dynamics compared to linear time series; therefore, $N I_{\alpha}$ was set at the $100 \cdot \alpha$-percentile of the distribution of NI over the surrogates, and the null hypothesis was rejected if $N I_{o}<N I_{\alpha}$. In the case of the IS and GLC indexes measuring the regularity of a time series or the amount of nonlinear correlations, the indexes are expected to increase in the presence of nonlinear dynamics compared to linear time series; therefore, $N I_{\alpha}$ was set at the $100 \cdot(1-\alpha)$-percentile of the distribution of $N I$ over the surrogates and the null hypothesis was rejected if $N I_{o}>$ $N I_{\alpha}$. In either case, rejection of the null hypothesis allows determining, individually for each subject, if the original value of the considered measure stands outside the $95 \%$ confidence interval of the surrogate distribution, thus detecting the presence of nonlinear dynamics.

\section{B. Quantification of nonlinear dynamics}

Surrogate time series were also exploited to quantify the "extent" of nonlinearity in each investigated HRV recoding, intended as the extent to which the original value of a measure deviates from its average value obtained from linear time series sharing the autocorrelation and amplitude distribution of the original series. This was performed comparing the index $N I_{o}$ computed on the original, possibly nonlinear time series, with the median $N I_{m}$ of its values computed on the set of surrogate time series. The difference with the median, defined as $\Delta N I=N I_{m}-N I_{o}$ in the case of the complexity index (i.e., when $N I=N C I$ ), and defined as $\Delta N I=N I_{o}-N I_{m}$ in the case of the two other indexes (i.e., when $N I=I S$ or when $N I=G L C$ ), was taken as a measure of the amount of nonlinearity in the observed time series. Moreover, an alternative measure accounting for the spread of the distribution of the nonlinearity measure was defined by normalizing the difference with the median to the standard deviation of the distribution of the measure over the surrogates [i.e., computing the ratio $\Delta N I / S D(N I)]$. Results for this standardized difference are reported in the supplementary material.

\section{PATIENTS, EXPERIMENTAL PROTOCOL, AND DATA ANALYSIS}

The time series analyzed in this study belong to an historical database collected to analyze the effects of aging and myocardial infarction on cardiovascular interactions. ${ }^{45}$ The database consists of heart rate variability measured in a group of 35 postacute myocardial infarction ( $A M I, 4$ female, $58.5 \pm 10.2$ years old) patients examined about 10 days after AMI and in two control groups formed by 12 old healthy subjects (Old, 9 female, $63.1 \pm 8.3$ years) and by 19 young healthy subjects (Young, 9 female, $25.0 \pm 2.6$ years). The Old subjects were matched in age with the post-AMI patients, and both groups were significantly older than the young subjects (two sample t-test on age distributions: $p=0.17$ Old vs AMI; $p<$ 0.00001 Old vs Young, AMI vs Young). Eight out of 35 post-AMI patients were initially under a beta-blocker therapy, but they discontinued the treatment two half-lives before the recording session. Control subjects were normotensive and free from any known disease based on anamnesis and physical examination at the time of the study.

After a period of $15 \mathrm{~min}$ for subject stabilization, the electrocardiogram (Siemens Mingograph, hardware bandpass filter $0.3-1000 \mathrm{~Hz}$, lead II ECG) was recorded for $10 \mathrm{~min}$ in the supine rest position, followed by $10 \mathrm{~min}$ of passive head-up tilt at $60^{\circ}$. All ECG signals were digitized with a 12 bit resolution and $1-\mathrm{KHz}$ sampling rate. After detecting the QRS complex on the ECG and locating the $\mathrm{R}$ apex through template matching, heart period variability was measured on a beat-to-beat basis calculating the sequence of the time intervals occurring between pairs of consecutive $R$ peaks (RR intervals). The series were then cleaned up from artifacts, windowed to $N=300$ points for each condition (rest, tilt) and detrended by a high-pass filter to fulfill stationarity criteria. ${ }^{44,66}$ Normalized time series were eventually obtained by subtracting the mean values and dividing by the standard deviation.

For each subject and condition, analysis of nonlinearity was performed using the three methods described in Sec. II and performing the tests described in Sec. III. NCI and IS indexes were computed using standard values for the free parameters of entropy estimators applied to short time series, ${ }^{48,58}$ namely, using $m=2$ values to approximate the past history of the process, setting a tolerance $r=0.2 \sigma$ to define similarity in sample entropy analysis (where $\sigma$ is the standard deviation of the series equal to 1 after normalization) and employing $k=10$ neighbors in the distance-based entropy estimations. Distances between patterns were obtained using the Eucilidean norm in the kernel estimator used to compute $\mathrm{NCI}^{51}$ and the maximum norm in the nearest-neighbor estimator used in IS. ${ }^{66}$ Moreover, to investigate the dependence on the type of entropy estimator and on the embedding dimension, the IS measure was computed also using the kernel estimator and varying the dimension in the range $m=(2,3,4)$. In the computation of the GLC index, taking into account the short size of the time series $(N=300)$ and to align with the other measures, we choose $\ell_{\max }=m=2$ to limit spurious results induced by the fact that the autocorrelation function tends to reach quickly the noise level.

For each assigned index (NCI, IS, GLC), the statistical significance of its changes across groups (Young, Old, AMI) and conditions (rest, tilt) was assessed by the two-way ANOVA, introducing the 
gender of the subjects as a categorical confounder variable in the test design. The assumption of Gaussianity was checked using the Jarque-Bera normality test, suggesting Gaussianity in the large majority of the distributions tested (15 out of 18 tests). Post hoc tests were performed using the two sample t-test with unequal variances to detect pairwise differences between groups (limited to the comparisons Young vs Old and Old vs AMI, with Bonferroni correction for this double comparison) and using the paired Student t-test to detect pairwise differences between the two conditions (rest vs tilt). We computed also the percentage of subjects belonging to each group for which the null hypothesis of linear Gaussian dynamics was rejected in the two conditions; then, statistically significant variations between two groups in a given condition were assessed using the chi-square test for proportions, while significant variations between conditions for a given group were assessed using the McNemar test for paired proportions.

\section{RESULTS}

Figure 1 reports an illustrative example of the application of the three considered nonlinear dynamic measures to individual HRV time series measured from one Young subject, one Old subject, and one $A M I$ patient in the two analyzed experimental conditions (rest, tilt). In each panel and for each of the two conditions, the individual value of the considered measure is plotted with a symbol, and the distribution of values obtained from the same measure over 100 surrogate time series is represented with an error bar (median and 5th --95 th percentiles); significant nonlinear dynamics are detected when the original value lies outside the surrogate distribution, while the extent of the deviation can be inferred comparing the original value and the surrogate median. Considering the two entropy measures, opposite response to the change in condition is observed consistently for the three cases, with lower values of NCI and higher values of IS measured during tilt compared to rest. On the contrary, moving from rest to tilt, the nonlinear dynamic measure based on GLC decreases slightly for the Young subject (circles), decreased more consistently for the Old subject (squares), and increases for the $A M I$ patient (triangles). Moreover, the comparison between the original value of a measure and its distribution on the surrogate time series reveals the different ability to detect nonlinear dynamics of the different measures. In particular, in both the experimental conditions, nonlinear dynamics are detected only by the information storage in the Young subject [Fig. 1(a)] and only by the Gaussian linear contrast method in the AMI patient [Fig. 1(c)], while all measures detect the presence of nonlinear dynamics in the Old subject [Fig. 1(b), NCI and IS in both conditions, and GLC only at rest].

Most of the trends observed for the representative subjects described above are reflected at the population level, as reported in Fig. 2, showing the distributions across subjects and conditions of the three nonlinear dynamic measures. The indexes based on conditional entropy and mutual information display opposite trends in response to the change of posture: the transition from rest to tilt is associated with a statistically significant decrease of the complexity index [NCI, Fig. 2(a)] in both Young $(p<0.0005)$ and AMI $(p=0.025)$ and a statistically significant increase of the information storage [IS, Fig. 2(b); $p<0.0005]$ in both Young $(p<0.0005)$ and $A M I$
( $p<0.0005)$, while no significant changes are detected for both measures in the Old group. Moreover, during tilt, NCI is significantly higher [Fig. 2(a); $p<0.0001$ ], and IS is significantly lower [Fig. 2(b); $p<0.0001$ ], in Old compared to Young. As to the GLC measure, it changes with the experimental condition in different ways for the different groups [Fig. 2(c)]: moving from rest to tilt, the measure decreases significantly in the Young subjects $(p=0.041)$, does not change significantly in the Old subjects and increases significantly in the $A M I$ patients $(p=0.023)$.

Figure 3 depicts the results of the analysis performed considering the deviation of each nonlinear dynamic measure from its median level assessed on linear Gaussian surrogates. We find that all three measures decrease significantly, in Young healthy subjects, with the transition from rest to tilt $(\triangle N C I: p=0.025 ; \Delta I S: p=0.024 ; \Delta I S$ : $p=0.01$ ), while no significant changes are observed for Old subjects and AMI patients [Figs. 3(a) and 3(b)]. It should be remarked that the assessment of the statistical significance reported for these cases may be influenced by deviations from normality, as the Jarque-Bera test did not reject the null hypothesis of joint Gaussianity for the rest and tilt distributions of the $\triangle I S$ and $\triangle G L C$ indexes evaluated in the Young subjects.

Figure 4 reports the relevance of nonlinear dynamics in each group and experimental condition, measured as the percentage of subjects for which the value of the considered nonlinear dynamic measure computed for the original RR series is deemed (with 5\% significance) as not drawn from the distribution of the index derived from the surrogate RR series. The conditional entropy measure is associated with nonlinear dynamics in less than half of the subjects in each group, as the NCI index is found below the 5th percentile of its surrogate distribution in $\sim 35 \%$ of Young subjects, $\sim 45 \%$ of Old subjects, and $\sim 25 \%$ of $A M I$ patients [with no substantial differences between conditions, Fig. 4(a)]. The mutual information measure detects a considerably higher percentage of nonlinear dynamics, as the IS index is found above the 95th percentile of its surrogate distribution in more than half of the subjects in all groups and conditions [Fig. 3(b)]. In the Young group, the IS index is larger than the significance threshold in $95 \%$ of the subjects at rest and in $\sim 70 \%$ of the subjects during tilt; in the Old and AMI groups, the index is significantly lower during both conditions [Fig. 4(b)]. The different detection rate of nonlinear dynamics exhibited by the NCI and IS measures is further investigated in the supplementary material, implementing a kernel-based estimation of IS. The Gaussian linear contrast approach detects nonlinear dynamics in $\approx 30 \%-60 \%$ of subjects in all groups and conditions [Fig. 4(c)]. Moving from rest to tilt, the number of subjects with nonlinear dynamics detected by the GLC measure decreases in Young, while it increases in Old and AMI.

\section{DISCUSSION}

The purpose of this study was to perform a comparative investigation of the aptitude of three recently proposed nonlinear dynamic measures [i.e., Normalized Complexity Index (NCI) recently defined as a refinement of the well known sample entropy measure, ${ }^{51}$ Information Storage (IS) implemented through nearest-neighbor estimation with bias compensation, ${ }^{15}$ and Gaussian Linear Contrast $\left.(\mathrm{GLC})^{10}\right]$ to quantify the presence and the extent of nonlinear dynamics in short-term recordings of HRV obtained under different 
(a)

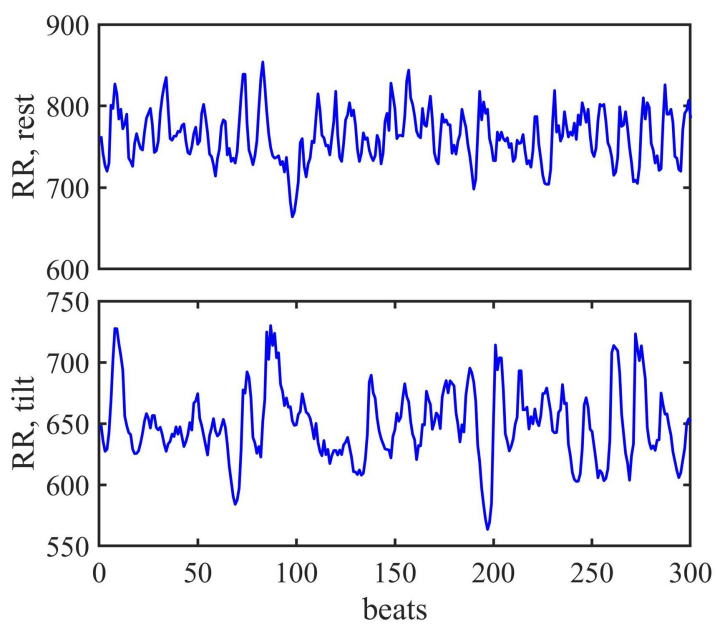

(b)

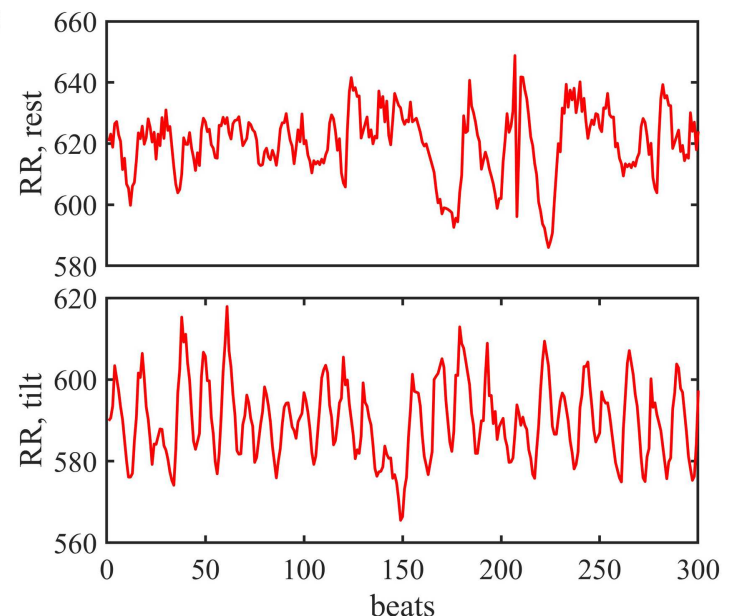

(c)

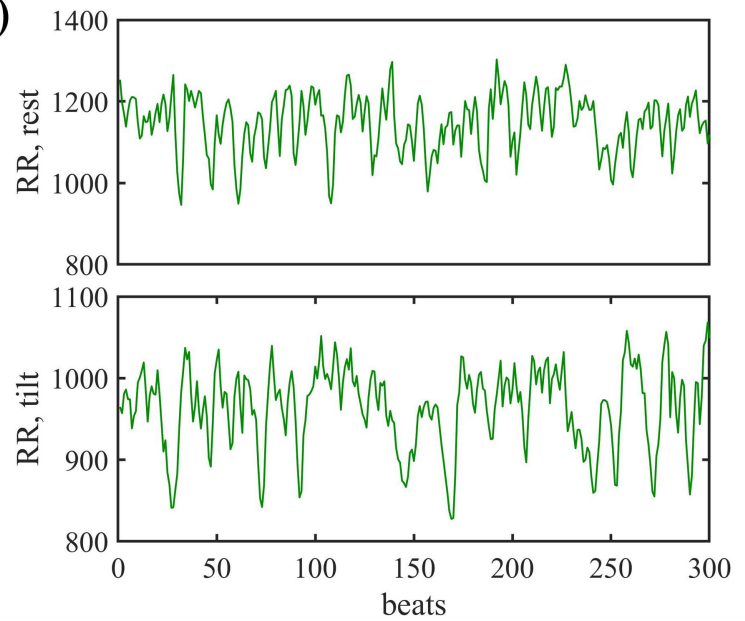

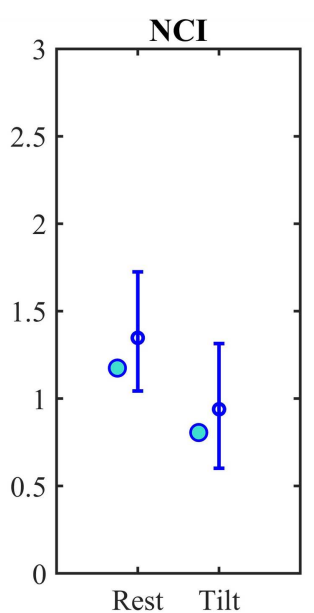
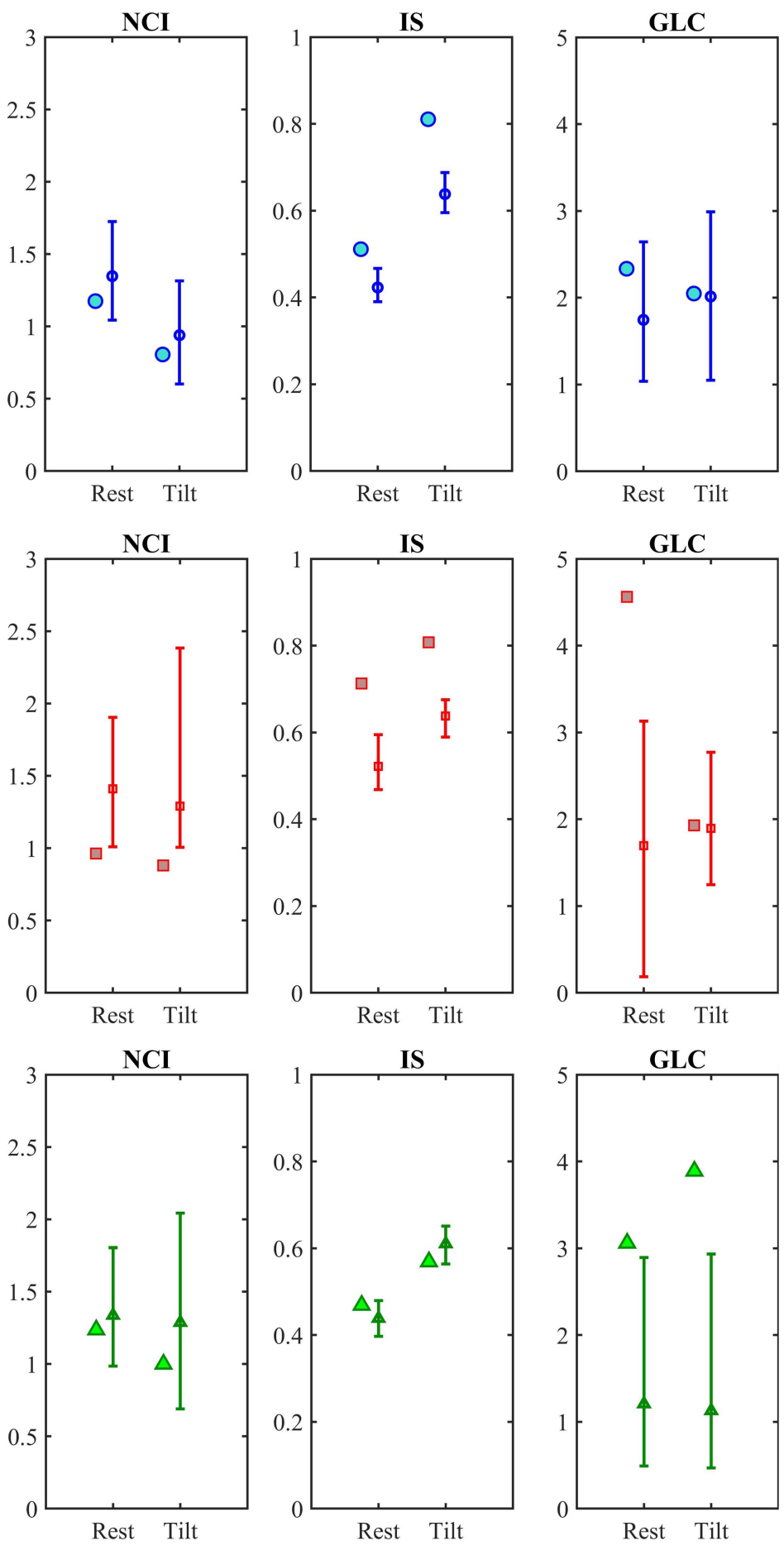

IS

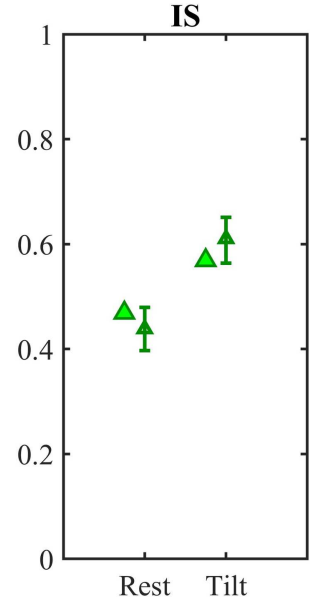

GLC

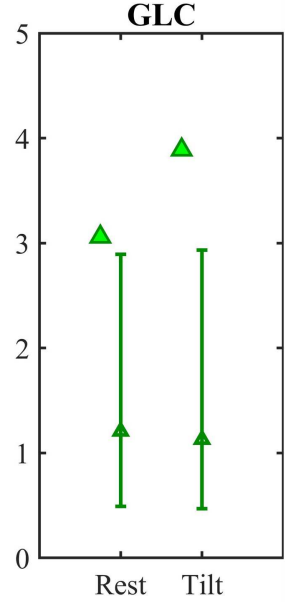

FIG. 1. Computation of nonlinear dynamic measures on heart period time series measured for a representative young subject [(a), blue], old subject [(b), red], and post-AMI patient [(c), green]. For each subject, the time series of the RR interval measured in the two experimental conditions are reported on the left (above: rest; below: tilt), and the values of the nonlinear dynamic measures obtained with the three considered methods (NCI: Normalized Complexity Index; IS: Information Storage; GLC: Gaussian Linear Contrast) are reported on the right (markers: original values; error bars: 5 th, 50th, and 95 th percentiles of the distribution over 100 surrogates). 

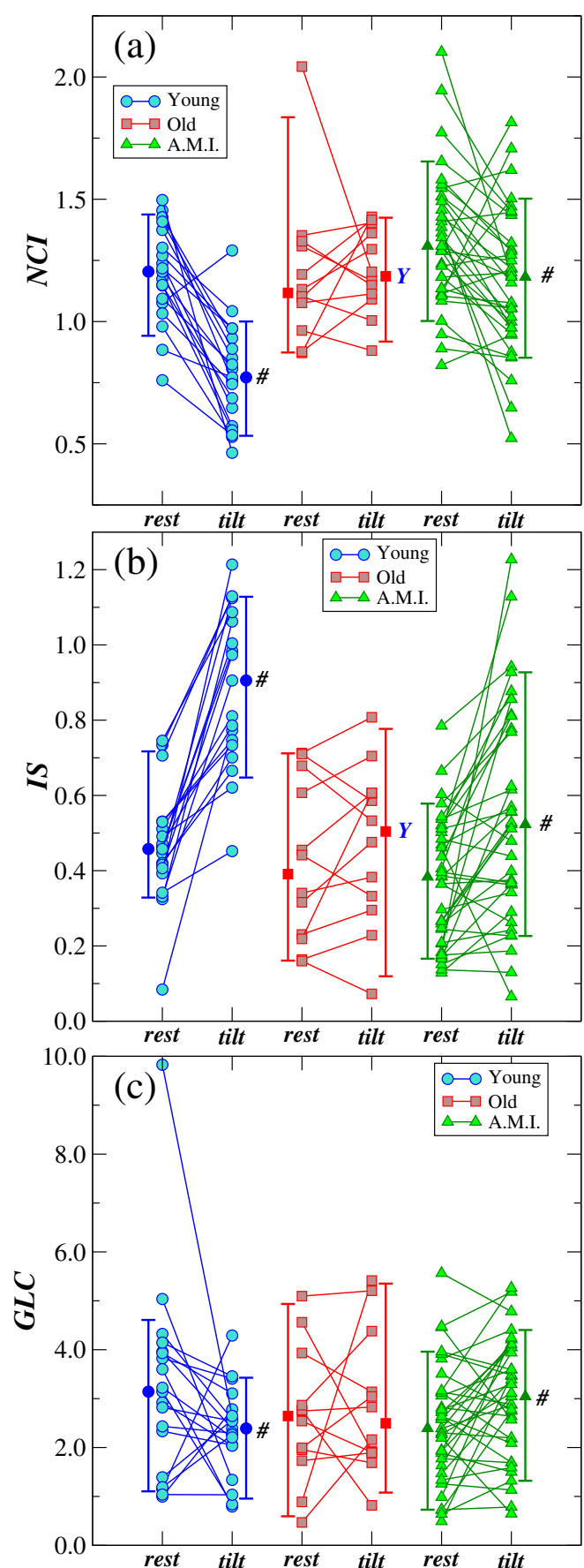

FIG. 2. Distribution of the three nonlinear dynamics measures [(a), normalized complexity index; (b) information storage; and (c) Gaussian linear contrast] over the HRV time series of Young subjects (blue circles), Old subjects (red squares), and post-AMI patients (green triangles) in the rest and tilt conditions, depicted as individual values (markers) and 5th, 50th, and 95th percentiles of the distributions across subjects (error bars). Symbols denote statistically significant changes between conditions (\#, rest vs tilt) or groups ( $Y$, Old vs Young); see text for details.
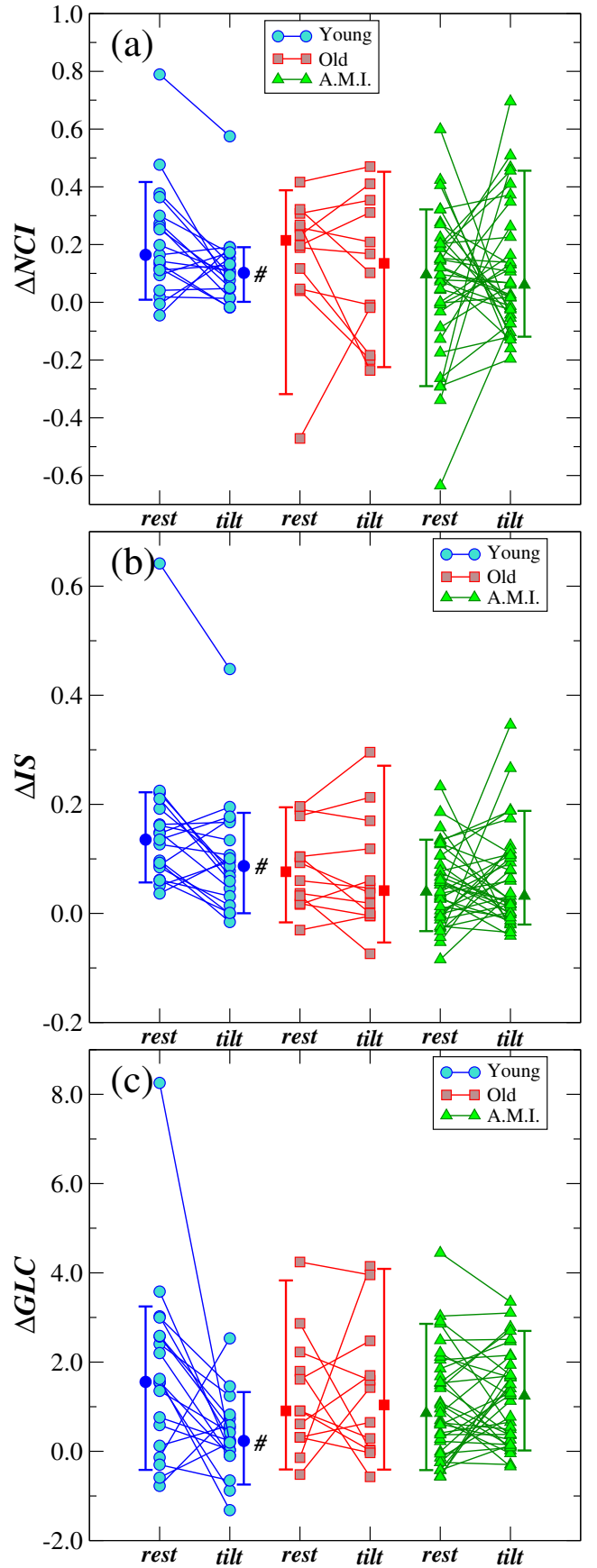

FIG. 3. Distribution of the difference with the median over surrogates of the three nonlinear dynamics measures [(a), normalized complexity index; (b) information storage; (c) Gaussian linear contrast) over the HRV time series of Young subjects (blue circles), Old subjects (red squares), and post-AMI patients (green triangles) in the rest and tilt conditions, depicted as individual values (markers) and 5th, 50th, and 95th percentiles of the distributions across subjects (error bars). The symbol \# denotes statistically significant changes between conditions (rest vs tilt); see text for details. 

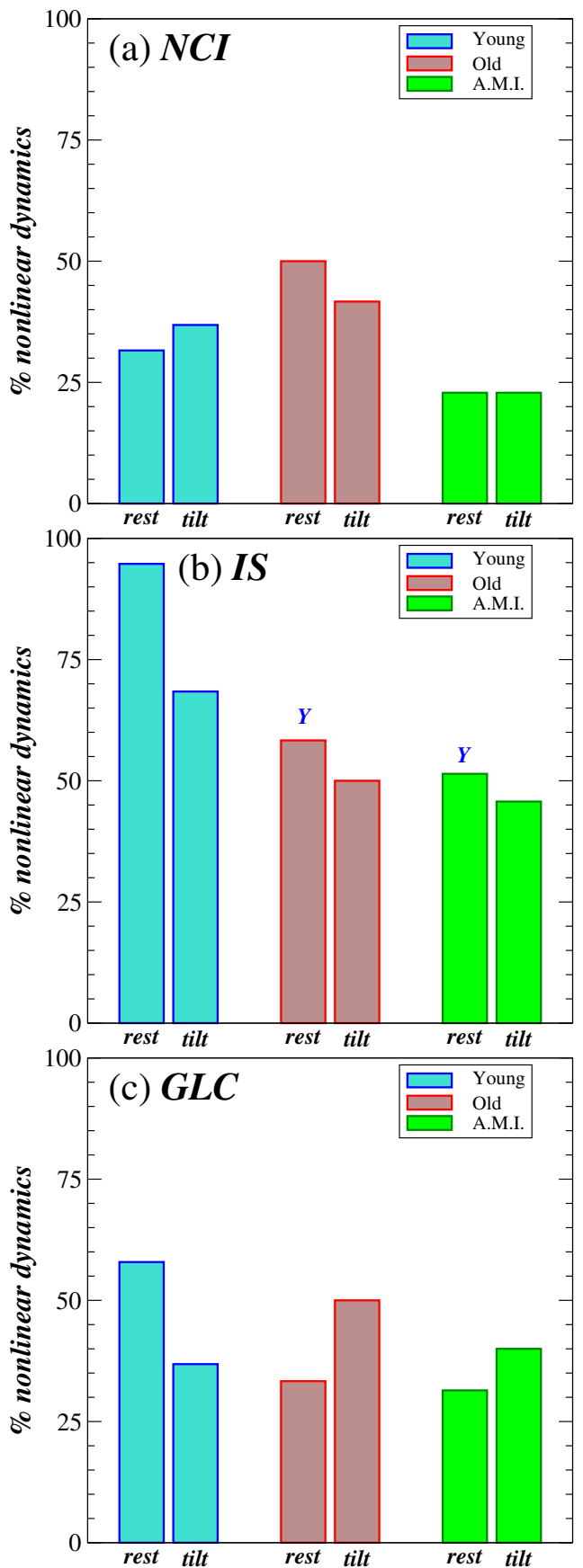

FIG. 4. Percentage of significant nonlinear dynamics obtained counting the subjects for which each nonlinear dynamic measure was significantly different for the original heart period than for the surrogate time series. Results are shown for each of the three proposed methods [(a), normalized complexity index; b, information storage; c, Gaussian linear contrast) applied to Young subjects (blue), Old subjects (red), and post-AMl patients (green) in the rest and tilt conditions. The symbol $Y$ denotes statistically significant changes between groups (Young vs Old); see text for details. physiopathological states. In a time series observed as a realization of a stochastic process, nonlinear dynamics are typically described as nonlinear correlations between time-lagged variables taken from the process. ${ }^{61}$ In our analysis, these correlations are detected directly in terms of mutual information between the present and the past samples of the process by the IS index, inversely in terms of conditional entropy of the present sample given in the past by the NCI index, or in terms of deviation of the estimated correlation from the value that would be expected in the case of linear correlations by the GLC index. Our results document that differences in the detection and quantification of nonlinearity emerge among the three measures, suggesting that a given nonlinear dynamic measure may be more or less sensitive to the detection of specific types of nonlinear dynamics depending on the properties of the measure and on the estimator adopted. Therefore, while some of the results are robust and suggest that distinct nonlinear dynamic structures may underlie the generation of HRV depending on the physiopathological condition under analysis, physiological interpretations should be provided with cautiousness.

A first result is the opposite variation exhibited by the indexes of conditional entropy and information storage when moving from rest to tilt or while comparing two groups [Figs. 2(a) and 2(b)]. This result, which is found consistently to also vary some analysis parameters such as the type of entropy estimator and the embedding dimension (see the supplementary material), can be explained considering that NCI and IS are related to each other as they reflect, respectively, the unpredictability and the predictability of the dynamics. ${ }^{66}$ The lower NCI and higher IS measured in response to tilt indicate higher predictability of HRV, likely associated to the activation of the sympathetic nervous system induced by the postural challenge. ${ }^{16,56}$ Such an activation seems less important in the old and post-AMI groups compared with the young subjects, as documented by the smaller variation of the indexes (though still statistically significant in $\mathrm{AMI}$ ) and by the higher $\mathrm{NCI} /$ lower IS seen during tilt in Old and AMI compared to Young. Confirming previous studies, ${ }^{39,45}$ these results suggest that aging and myocardial infarction are associated with higher sympathetic tone and reduced capability to cope with the postural challenge with further sympathetic activation.

On the other hand, the trends displayed by the GLC measure [Fig. 2(c)] are in agreement with those of the conditional entropy in the young subjects (both GLC and NCI decrease with tilt) and with those of the information storage in the AMI patients (both GLC and IS increase with tilt). The different behavior of the GLC index can be explained by considering that this index reflects the extent to which the correlations of the time series deviate from those expected in the linear Gaussian case, ${ }^{10}$ and thus, it is not dependent on the extent of linear correlations within the observed time series. As such, GLC should be interpreted as a direct measure of nonlinearity rather than as a regularity index. This is confirmed by the consistent changes between conditions displayed by the absolute values of GLC and by the difference between the index and the median value of its surrogate counterparts [Fig. 2(c) vs Fig. 3(c)]. On the contrary, IS is a regularity measure, which accounts for both linear and nonlinear correlations, and its increase with tilt is mainly driven by the enhancement of linear HRV correlations. In fact, when the effects of linear correlations are removed by subtracting the median on the surrogates, the behavior of IS becomes more similar to that of GLC [Figs. 3(b) and 3(c)]. This similar behavior is observed consistently 
for all three measures when nonlinear HRV dynamics were assessed computing the deviation of each measure from the median level of its surrogate distribution [Figs. 3(a)-3(c)]. The difference with the surrogate median reveals indeed that, in the young healthy subjects, the transition from rest to tilt is associated with a decreased degree of nonlinearity, and this result is in agreement with the observation that nonlinear dynamics are reduced in the presence of an increased sympathetic activity. ${ }^{53,57}$ Another peculiar result is the increased contribution of nonlinear dynamics to HRV measured during tilt in the post-AMI patients [Fig. 2(c)]. This finding is novel and unexpected and may reveal that a distinct type of nonlinearity takes place when the orthostatic stress is delivered in the presence of higher sympathetic tone.

In spite of the similar trends observed for the absolute values and for the deviation from the surrogate median value of NCI and IS [Figs. 3(a) and 3(b)], the two information measures exhibit different percentages of significant nonlinearity in the various conditions [Figs. 4(a) and 4(b)]. In agreement with previous studies assessing complexity through prediction measures, ${ }^{53,57}$ the amount of nonlinear dynamics detected by the complexity measure based on local sample entropy was small in the young healthy subject at rest and did not change significantly with the sympathetic activation induced by tilt or related to age and pathology [Fig. 4(a)]. On the contrary, using a regularity measure based on information storage, nonlinear dynamics were found consistently in the young subjects at rest, and their incidence decreased significantly with postural stress and in the old and post-AMI groups [Fig. 4(b)]. This finding may reflect the fact that the spontaneous cardiovascular regulation occurs through a variety of nonlinear mechanisms (e.g., saturation of receptors, effects of the respiratory centers at the brain stem level, interaction between sympathetic and parasympathetic nervous systems, etc.) in resting conditions, ${ }^{31}$ and the rise of a specific oscillatory component (i.e., the low frequency one related to sympathetic activation) tends to simplify the dynamics reducing nonlinear components. The reduction of nonlinear dynamics with the tilt-induced sympathetic activation is confirmed (though to a lower extent) by the test using the GLC measure [Fig. 4(c)]. The same test, however, indicates a tendency to increase the rate of detection of nonlinear dynamics with tilt in the old subjects and AMI patients. This could suggest that mechanisms more complex than a pure sympathetic activation are triggered by the orthostatic stress delivered in the elderly and pathological states. ${ }^{40}$

However, more methodological factors might be responsible for the disparity of the conclusions drawn by the exploited markers. In a previous study, ${ }^{52}$ different conclusions about HRV nonlinear dynamics were drawn using different nonlinearity measures (based on nonlinear prediction and time irreversibility) in fetal HRV recordings as well as in adults during graded head-up tilt. In particular, the different responses to tilt documented by Porta et al. ${ }^{52}$ using nonlinear prediction and time irreversibility are comparable to those observed here using the IS and GLC indexes. The different rates of detection of nonlinearity were explained by Porta et al. ${ }^{52}$ in terms of the different time scales spanned by the measures employed. However, this interpretation should not hold in our case since all measures work in the same low dimensional embedding space $(m=2$ in this study). The choice $m=2$, which corresponds to a reconstructed state space defining vectors $\left(S_{n}, S_{n-1}\right)$, reflects a typical setting in shortterm HRV analysis. ${ }^{16,51,56,66}$ While this choice prevents the detection of higher dimensional dynamics, which may occur in HRV, in shortterm analysis, it is recommended to keep reliability of the estimated entropy measures in the presence of short data sequences. ${ }^{47}$ Here, we observed that the adopted entropy measures bring results that are robust against changes of this parameter in the range $m=(2,3,4)$ (see the supplementary material).

A difference between the information approach and the Gaussian linear contrast method is that NCI and IS are obtained aggregating all time lags in the computation of the measure, while GLC results from analyses performed individually for each lag and then aggregated in the final measure. In addition, the performance of GLC might be affected by the comparison with surrogates that might have amplified eventual residual departures from Gaussianity present in the surrogate data due to finite size effects. Moreover, the dependence of the cell size on the parameters set for the analysis (i.e., respectively, tolerance and number of nearest neighbors) might also have played a role. In order to better elucidate the nature of the observed differences and the capability of the various measures to detect different types of nonlinear dynamics, future studies should consider extension of these measures where longer temporal scales can be explored (e.g., analyzing longer stationary recordings and/or employing methods for dimensionality reduction ${ }^{16}$ ), and deviations of the estimator specific parameters from their nominal typical value are investigated (e.g., for the information measures, the parameter setting the size of the cell used in the multidimensional space to estimate probabilities $^{15,58}$ ).

As for the differences observed between NCI and IS, our additional analysis comparing kernel and nearest-neighbor estimates of the IS index (see the supplementary material) suggests that the type of estimator can be crucial in the detection of nonlinear dynamics. Indeed, when IS is computed via kernel estimates rather than via nearest neighbors, the percentage of significant nonlinear dynamics decreases, becoming comparable to that detected by the NCI index, which is also grounded on kernel-based entropy estimation. This suggests that different coarse graining approaches using, respectively, equal vs different cell size ${ }^{57}$ may result in a different sensitivity as regards the detection of nonlinear dynamics. In particular, the nearest-neighbor estimator turns out to be a better estimator for the information measures, as it shows higher sensitivity to the detection of nonlinear dynamics. Another methodological aspect that can explain the difference is the fact that nearest-neighbor estimation is accompanied by a distance-projection approach such that the cell size actually varies only while estimating entropies in the highest dimension, while it is kept constant (while allowing the number of neighbors to vary) in the lower dimensional spaces. ${ }^{33}$ Thus, we suggest that the bias of entropies computed in spaces of different dimension plays a role in the detection of nonlinear dynamics. This aspect should be investigated in more methodological studies involving simulations of linear and nonlinear dynamic systems.

Finally, we mention as possible limitations of the present study some characteristics of the analyzed database. ${ }^{45}$ The three analyzed groups are not homogeneous in the gender distribution (males are prevalent in $A M I$, females are prevalent in $\mathrm{Old}$, and the gender is balanced in Young), and this may have an impact on the results as it is known that gender has a substantial effect on heart rate variability. ${ }^{63,67}$ Moreover, residual effects of the treatment with betablockers may be present in the AMI patients and have an effect on the dynamics 
analyzed. The results reported in this study should be confirmed on other databases with balanced gender distribution and the absence of fully discontinued treatment after myocardial infarction.

\section{SUPPLEMENTARY MATERIAL}

See the supplementary material for additional analysis, illustrating the dependence of the measure of information storage on the embedding dimension and on the type of the estimator used to compute entropies.

\section{ACKNOWLEDGMENTS}

L.F. acknowledges financial support by PRIN (Progetti di Ricerca di Interesse Nazionale) 2017, Ministero dell'Istruzione, dell'Università e della Ricerca (MIUR), PRJ-0167, "Stochastic forecasting in complex systems", University of Palermo, Italy. P.C. and P.B.-G. acknowledge financial support 868 by the Consejería de Conocimiento, Investigación y Universidad, 869 Junta de Andalucía, and the European Regional Development Fund 870 (ERDF), Ref. Nos. SOMM17/6105/UGR, FQM-362, and FQM-7964.

\section{REFERENCES}

${ }^{1}$ U. Rajendra Acharya, K. Paul Joseph, N. Kannathal, C. M. Lim, and J. S. Suri, "Heart rate variability: A review," Med. Biol. Eng. Comput. 44(12), 1031-1051 (2006).

${ }^{2}$ S. Akselrod, D. Gordon, F. Andrew Ubel, D. C. Shannon, A. C. Berger, and R. J. Cohen, "Power spectrum analysis of heart rate fluctuation: A quantitative probe of beat-to-beat cardiovascular control," Science 213(4504), 220-222 (1981).

${ }^{3}$ Y. Bai, K. L. Siu, S. Ashraf, L. Faes, G. Nollo, and K. H. Chon, "Nonlinear coupling is absent in acute myocardial patients but not healthy subjects," Am. J. Physiol. Heart Circ. Physiol. 295(2), H578-H586 (2008).

${ }^{4}$ G. Baselli, S. Cerutti, S. Civardi, F. Lombardi, A. Malliani, M. Merri, M. Pagani, and G. Rizzo, "Heart rate variability signal processing: A quantitative approach as an aid to diagnosis in cardiovascular pathologies," Int. J. Biomed. Comput. 20(1-2), 51-70 (1987).

${ }^{5}$ P. Bernaola-Galván, P. Ch. Ivanov, L. A. Nunes Amaral, and H. Eugene Stanley, "Scale invariance in the nonstationarity of human heart rate," Phys. Rev. Lett. 87(16), 168105 (2001).

${ }^{6}$ P. A. Bernaola-Galván, M. Gómez-Extremera, A. Ramón Romance, and P. Carpena, "Correlations in magnitude series to assess nonlinearities: Application to multifractal models and heartbeat fluctuations," Phys. Rev. E 96(3), 032218 (2017).

${ }^{7}$ G. G. Berntson, J. Thomas Bigger, Jr., D. L. Eckberg, P. Grossman, P. G. Kaufmann, M. Malik, H. N. Nagaraja, S. W. Porges, J. Philip Saul, P. H. Stone et al., "Heart rate variability: Origins, methods, and interpretive caveats," Psychophysiology 34(6), 623-648 (1997).

${ }^{8}$ J. Thomas Bigger, Jr., J. L. Fleiss, R. C. Steinman, L. M. Rolnitzky, R. E. Kleiger, and J. N. Rottman, "Frequency domain measures of heart period variability and mortality after myocardial infarction," Circulation 85(1), 164-171 (1992).

${ }^{9}$ M. C. Cario and B. L. Nelson, "Autoregressive to anything: Time-series input processes for simulation," Oper. Res. Lett. 19(2), 51-58 (1996).

${ }^{10}$ P. Carpena, P. A. Bernaola-Galván, M. Gómez-Extremera, and A. V. Coronado, "Transforming Gaussian correlations. Applications to generating longrange power-law correlated time series with arbitrary distribution," e-print arXiv:1909.01725 (2019).

${ }^{11} \mathrm{H}$. Chen, "Initialization for norta: Generation of random vectors with specified marginals and correlations," INFORMS J. Comput. 13(4), 312-331 (2001).

${ }^{12}$ K. Chua Chua, V. Chandran, U. R. Acharya, and C. M. Lim, "Cardiac state diagnosis using higher order spectra of heart rate variability," J. Med. Eng. Technol. 32(2), 145-155 (2008).
${ }^{13} \mathrm{M}$. A. Cohen and J. Andrew Taylor, "Short-term cardiovascular oscillations in man: Measuring and modelling the physiologies," J. Physiol. (Lond.) 542(3), 669-683 (2002).

${ }^{14}$ M. Costa, I. R. Pimentel, T. Santiago, P. Sarreira, J. Melo, and E. Ducla-Soares, "No evidence of chaos in the heart rate variability of normal and cardiac transplant human subjects," J. Cardiovasc. Electrophysiol. 10(10), 1350-1357 (1999).

${ }^{15}$ L. Faes, D. Kugiumtzis, G. Nollo, F. Jurysta, and D. Marinazzo, "Estimating the decomposition of predictive information in multivariate systems," Phys. Rev. E 91(3), 032904 (2015).

${ }^{16} \mathrm{~L}$. Faes and A. Porta, "Conditional entropy-based evaluation of information dynamics in physiological systems," in Directed Information Measures in Neuroscience (Springer, 2014), pp. 61-86.

${ }^{17}$ J.-O. Fortrat, Y. Yamamoto, and R. L. Hughson, "Respiratory influences on nonlinear dynamics of heart rate variability in humans," Biol. Cybern. 77(1), 1-10 (1997).

${ }^{18}$ R. Freeman, J. Philip Saul, M. S. Roberts, R. D. Berger, C. Broadbridge, and R. J. Cohen, "Spectral analysis of heart rate in diabetic autonomic neuropathy: A comparison with standard tests of autonomic function," Arch. Neurol. 48(2), 185-190 (1991).

${ }^{19}$ L. Glass, "Introduction to controversial topics in nonlinear science: Is the normal heart rate chaotic?," Chaos 19(2), 028501 (2009).

${ }^{20}$ M. Gómez-Extremera, P. A. Bernaola-Galván, S. Vargas, J. Benítez-Porres, P. Carpena, and A. Ramón Romance, "Differences in nonlinear heart dynamics during rest and exercise and for different training," Physiol. Meas. 39(8), 084008 (2018).

${ }^{21}$ R. B. Govindan, K. Narayanan, and M. S. Gopinathan, "On the evidence of deterministic chaos in ECG: Surrogate and predictability analysis," Chaos 8(2), 495-502 (1998).

${ }^{22}$ A. R. Gujjar, T. N. Sathyaprabha, D. Nagaraja, K. Thennarasu, and N. Pradhan, "Heart rate variability and outcome in acute severe stroke," Neurocrit. Care 1(3), 347-353 (2004).

${ }^{23}$ H. V. Huikuri, T. H. Mäkikallio, C.-K. Peng, A. L. Goldberger, U. Hintze, and M. Møller, "Fractal correlation properties of RR interval dynamics and mortality in patients with depressed left ventricular function after an acute myocardial infarction," Circulation 101(1), 47-53 (2000).

${ }^{24}$ P. Ch. Ivanov, L. A. Nunes Amaral, A. L. Goldberger, S. Havlin, M. G. Rosenblum, Z. R. Struzik, and H. Eugene Stanley, "Multifractality in human heartbeat dynamics,” Nature 399(6735), 461-465 (1999).

${ }^{25}$ N. Iyengar, C. K. Peng, R. Morin, A. L. Goldberger, and L. A. Lipsitz, "Age-related alterations in the fractal scaling of cardiac interbeat interval dynamics," Am. J. Physiol. Regul. Integr. Comp. Physiol. 271(4), R1078-R1084 (1996).

${ }^{26}$ J. K. Kanters, M. V. Hojgaard, E. Agner, and N. H. Holstein-Rathlou, "Influence of forced respiration on nonlinear dynamics in heart rate variability," Am. J. Physiol. Regul. Integr. Comp. Physiol. 272(4), R1149-R1154 (1997).

${ }^{27}$ J. K. Kanters, M. V. Højgaard, E. Agner, and N.-H. Holstein-Rathlou, "Short-and long-term variations in non-linear dynamics of heart rate variability," Cardiovasc. Res. 31(3), 400-409 (1996).

${ }^{28}$ J. K. Kanters, N.-H. Holstein-Rathlou, and E. Agner, "Lack of evidence for lowdimensional chaos in heart rate variability," J. Cardiovasc. Electrophysiol. 5(7), 591-601 (1994)

${ }^{29}$ D. T. Kaplan, M. I. Furman, S. M. Pincus, S. M. Ryan, L. A. Lipsitz, and A. L. Goldberger, "Aging and the complexity of cardiovascular dynamics," Biophys. J. 59(4), 945-949 (1991).

${ }^{30}$ R. Karasik, N. Sapir, Y. Ashkenazy, P. Ch. Ivanov, I. Dvir, P. Lavie, and S. Havlin, "Correlation differences in heartbeat fluctuations during rest and exercise," Phys. Rev. E 66(6), 062902 (2002).

${ }^{31}$ H. P. Koepchen, "Physiology of rhythms and control systems: An integrative approach," in Rhythms in Physiological Systems (Springer, 1991), pp. 3-20.

${ }^{32}$ L. F. Kozachenko and N. N. Leonenko, "Sample estimate of the entropy of a random vector," Probl. Peredachi Inf. 23(2), 9-16 (1987).

${ }^{33}$ A. Kraskov, H. Stögbauer, and P. Grassberger, "Estimating mutual information," Phys. Rev. E 69(6), 066138 (2004).

${ }^{34}$ D. Kugiumtzis, "Surrogate data test for nonlinearity including nonmonotonic transforms," Phys. Rev. E 62(1), R25 (2000).

${ }^{35}$ D. Kugiumtzis, "Statically transformed autoregressive process and surrogate data test for nonlinearity,” Phys. Rev. E 66(2), 025201 (2002). 
${ }^{36}$ D. Kugiumtzis and E. Bora-Senta, "Normal correlation coefficient of non-normal variables using piece-wise linear approximation," Comput. Stat. 25(4), 645-662 (2010).

${ }^{37}$ S. Ted Li and J. L. Hammond, "Generation of pseudorandom numbers with specified univariate distributions and correlation coefficients," IEEE Trans. Syst. Man. Cybern. SMC-5(5), 557-561 (1975).

${ }^{38}$ J. T. Lizier, M. Prokopenko, and A. Y. Zomaya, "Local measures of information storage in complex distributed computation," Inf. Sci. 208, 39-54 (2012).

${ }^{39}$ F. Lombardi, G. Sandrone, S. Pernpruner, R. Sala, M. Garimoldi, S. Cerutti, G. Baselli, M. Pagani, and A. Malliani, "Heart rate variability as an index of sympathovagal interaction after acute myocardial infarction," Am. J. Cardiol. 60(16), 1239-1245 (1987).

${ }^{40} \mathrm{H}$. Luukinen, K. Koski, P. Laippala, and K. E. J. Airaksinen, "Orthostatic hypotension and the risk of myocardial infarction in the home-dwelling elderly," J. Intern. Med. 255(4), 486-493 (2004).

${ }^{41}$ M. Malik and A. John Camm, "Heart rate variability," Clin. Cardiol. 13(8), 570-576 (1990).

${ }^{42}$ N. Montano, T. Gnecchi Ruscone, A. Porta, F. Lombardi, M. Pagani, and A. Malliani, "Power spectrum analysis of heart rate variability to assess the changes in sympathovagal balance during graded orthostatic tilt," Circulation 90(4), 1826-1831 (1994)

${ }^{43}$ R. B. Nelsen, An Introduction to Copulas (Springer Science \& Business Media, 2007).

${ }^{44}$ G. Nollo, L. Faes, B. Pellegrini, A. Porta, and R. Antolini, "Synchronization index for quantifying nonlinear causal coupling between RR interval and systolic arterial pressure after myocardial infarction," in Computers in Cardiology 2000 (Cat. 00CH37163) (IEEE, 2000), Vol. 27, pp. 143-146.

${ }^{45}$ G. Nollo, L. Faes, A. Porta, B. Pellegrini, F. Ravelli, M. Del Greco, M. Disertori, and R. Antolini, "Evidence of unbalanced regulatory mechanism of heart rate and systolic pressure after acute myocardial infarction," Am. J. Physiol. Heart Circ. Physiol. 283(3), H1200-H1207 (2002).

${ }^{46}$ C.-K. Peng, S. Havlin, H. Eugene Stanley, and A. L. Goldberger, "Quantification of scaling exponents and crossover phenomena in nonstationary heartbeat time series," Chaos 5(1), 82-87 (1995).

${ }^{47} \mathrm{~S}$. M. Pincus, "Approximate entropy as a measure of system complexity," Proc. Natl. Acad. Sci. U.S.A. 88(6), 2297-2301 (1991).

${ }^{48}$ S. M. Pincus and A. L. Goldberger, "Physiological time-series analysis: What does regularity quantify?,” Am. J. Physiol. Heart Circ. Physiol. 266(4), H1643-H1656 (1994).

${ }^{49}$ A. Porta, G. Baselli, D. Liberati, N. Montano, C. Cogliati, T. Gnecchi-Ruscone, A. Malliani, and S. Cerutti, "Measuring regularity by means of a corrected conditional entropy in sympathetic outflow," Biol. Cybern. 78(1), 71-78 (1998).

${ }^{50}$ A. Porta, L. Faes, M. Masé, G. D’Addio, G. D. Pinna, R. Maestri, N. Montano, R. Furlan, S. Guzzetti, G. Nollo et al., "An integrated approach based on uniform quantization for the evaluation of complexity of short-term heart period variability: Application to 24h Holter recordings in healthy and heart failure humans," Chaos 17(1), 015117 (2007)

${ }^{51}$ A. Porta, V. Bari, B. De Maria, B. Cairo, E. Vaini, M. Malacarne, M. Pagani, and D. Lucini, "On the relevance of computing a local version of sample entropy in cardiovascular control analysis," IEEE Trans. Biomed. Eng. 66(3), 623-631 (2019). ${ }^{52}$ A. Porta, V. Bari, A. Marchi, B. De Maria, D. Cysarz, P. Van Leeuwen, A. Takahashi, A. M. Catai, and T. Gnecchi-Ruscone, "Complexity analyses show two distinct types of nonlinear dynamics in short heart period variability recordings," Front. Physiol. 6, 71 (2015)

${ }^{53}$ A. Porta, G. Baselli, S. Guzzetti, M. Pagani, A. Malliani, and S. Cerutti, "Prediction of short cardiovascular variability signals based on conditional distribution," IEEE Trans. Biomed. Eng. 47(12), 1555-1564 (2000).

${ }^{54}$ A. Porta, K. R. Casali, A. G. Casali, T. Gnecchi-Ruscone, E. Tobaldini, N. Montano, S. Lange, D. Geue, D. Cysarz, and P. Van Leeuwen, "Temporal asymmetries of short-term heart period variability are linked to autonomic regulation," Am. J. Physiol. Regul. Integr. Comp. Physiol. 295(2), R550-R557 (2008).

${ }^{55}$ A. Porta, L. Faes, A. Marchi, V. Bari, B. De Maria, S. Guzzetti, R. Colombo, and F. Raimondi, "Disentangling cardiovascular control mechanisms during headdown tilt via joint transfer entropy and self-entropy decompositions," Front. Physiol. 6, 301 (2015).

${ }^{56}$ A. Porta, T. Gnecchi-Ruscone, E. Tobaldini, S. Guzzetti, R. Furlan, and N. Montano, "Progressive decrease of heart period variability entropy-based complexity during graded head-up tilt," J. Appl. Physiol. Respir. Environ. Exerc. Physiol. 103(4), 1143-1149 (2007).

${ }^{57}$ A. Porta, S. Guzzetti, R. Furlan, T. Gnecchi-Ruscone, N. Montano, and A. Malliani, "Complexity and nonlinearity in short-term heart period variability: Comparison of methods based on local nonlinear prediction," IEEE Trans. Biomed. Eng. 54(1), 94-106 (2007).

${ }^{58} \mathrm{~J}$. S. Richman and J. Randall Moorman, "Physiological time-series analysis using approximate entropy and sample entropy," Am. J. Physiol. Heart Circ. Physiol. 278(6), H2039-H2049 (2000).

${ }^{59}$ T. Schreiber and A. Schmitz, "Improved surrogate data for nonlinearity tests," Phys. Rev. Lett. 77(4), 635 (1996)

${ }^{60}$ P. K. Stein, P. P. Domitrovich, H. V. Huikuri, R. E. Kleiger, and C. Investigators, "Traditional and nonlinear heart rate variability are each independently associated with mortality after myocardial infarction," J. Cardiovasc. Electrophysiol. 16(1), 13-20 (2005).

${ }^{61}$ J. Theiler, S. Eubank, A. Longtin, B. Galdrikian, and J. Doyne Farmer, "Testing for nonlinearity in time series: The method of surrogate data," Physica D 58(1-4), 77-94 (1992).

${ }^{62}$ Z. Visnovcova, M. Mestanik, M. Javorka, D. Mokra, M. Gala, A. Jurko, A. Calkovska, and I. Tonhajzerova, "Complexity and time asymmetry of heart rate variability are altered in acute mental stress," Physiol. Meas. 35(7), 1319 (2014).

${ }^{63}$ A. Voss, R. Schroeder, A. Heitmann, A. Peters, and S. Perz, "Short-term heart rate variability-Influence of gender and age in healthy subjects," PLOS One 10(3), e0118308 (2015).

${ }^{64}$ A. Voss, S. Schulz, R. Schroeder, M. Baumert, and P. Caminal, "Methods derived from nonlinear dynamics for analysing heart rate variability," Philos. Trans. R. Soc. A Math. Phys. Eng. Sci. 367(1887), 277-296 (2008).

${ }^{65} \mathrm{M}$. Wibral, J. T. Lizier, S. Vögler, V. Priesemann, and R. Galuske, "Local active information storage as a tool to understand distributed neural information processing," Front. Neuroinformatics 8, 1 (2014).

${ }^{66}$ W. Xiong, L. Faes, and P. Ch. Ivanov, "Entropy measures, entropy estimators, and their performance in quantifying complex dynamics: Effects of artifacts, nonstationarity, and long-range correlations," Phys. Rev. E 95(6), 062114 (2017).

${ }^{67} \mathrm{~J}$. Zhang, "Effect of age and sex on heart rate variability in healthy subjects," J. Manipulative Physiol. Ther. 30(5), 374-379 (2007). 